\title{
miR-128 Is Implicated in Stress Responses by Targeting MAFG in Skeletal Muscle Cells
}

\author{
Rocco Caggiano, ${ }^{1}$ Fabio Cattaneo, ${ }^{1,2}$ Ornella Moltedo, ${ }^{3}$ Giovanni Esposito, ${ }^{2}$ Cinzia Perrino, ${ }^{2}$ \\ Bruno Trimarco, ${ }^{2}$ Rosario Ammendola, ${ }^{1}$ and Raffaella Faraonio ${ }^{1,4}$ \\ ${ }^{1}$ Dipartimento di Medicina Molecolare e Biotecnologie Mediche, Università di Napoli Federico II, Napoli, Italy \\ ${ }^{2}$ Dipartimento di Scienze Biomediche Avanzate, Università di Napoli Federico II, Napoli, Italy \\ ${ }^{3}$ Dipartimento di Farmacia, Università degli Studi di Salerno, Fisciano, Salerno, Italy \\ ${ }^{4}$ CEINGE-Biotecnologie Avanzate s.c. a r.l, Napoli, Italy
}

Correspondence should be addressed to Raffaella Faraonio; faraonio@dbbm.unina.it

Received 4 April 2017; Revised 30 June 2017; Accepted 18 July 2017; Published 12 September 2017

Academic Editor: Jaideep Banerjee

Copyright ( 2017 Rocco Caggiano et al. This is an open access article distributed under the Creative Commons Attribution License, which permits unrestricted use, distribution, and reproduction in any medium, provided the original work is properly cited.

MAFG (v-Maf avian musculoaponeurotic fibrosarcoma oncogene homolog G) is a bZIP-type transcriptional regulator that belongs to the small MAF (sMAFs) protein family. By interacting with other bZIP transcription factors, sMAFs can form homo- and heterodimers governing either repressive or activating transcriptional functions. As heterodimeric partner of Nrf2, MAFG positively influences the ARE-dependent antioxidant/xenobiotic pathways, at least in condition of a correct MAFG:Nrf2 balance. MicroRNAs (miRs) participate to different regulatory networks being involved as fine-tuning regulators of gene expression. However, the connections between cellular surveillance to stresses mediated by MAFG:Nrf2 and miR regulations are not well understood. Here, we explored the impact of miR-128 in expression of genes related to stress response. Bioinformatic predictions coupled with functional analysis revealed the presence of miR-128 binding site in the 3'UTR of MAFG. Ectopic miR-128 expression correlated with reduced expression of endogenous MAFG-dependent genes and negatively affected ARE-mediated molecular phenotype based on Nrf2 activity. Indeed, miR-128 impairs redox-dependent pathways induced in response to oxidative stress. Moreover, in condition of hypoxia, MAFG induction correlated with reduced levels of miR-128. This lead to increased mRNA levels of HMOX-1 and $\mathrm{x}$-CT for blunting stress. Overall, these findings identify MAFG as novel direct target of miR-128.

\section{Introduction}

In response to oxidative and xenobiotic stresses, cells activate numerous defense systems associated with both enzymatic and not enzymatic activities. The events underlying these redox-related responses are accomplished by a tight regulation of gene expression patterns involving multilayered regulatory mechanisms $[1,2]$. Two transcription factors shown to be highly involved in the regulation of numerous antioxidant and detoxifying genes at transcriptional levels are Nrf2 [nuclear factor (erythroid-derived 2)-like 2] [3-5] and MAFG (v-Maf avian musculoaponeurotic fibrosarcoma oncogene homolog G) [6]. In particular, in the form of heterodimer, the complex Nrf2:MAFG binds to and activates the transcription of antioxidant/xenobiotic genes harboring antioxidant responsive elements (ARE)/electrophile responsive elements (core ARE: TGACNNNGC), located in their transcription regulatory sequences $[7,8]$.

The proteins of the MAF family, described for the first time as a viral oncogene in their prototype $\mathrm{v}$-Maf, are transcription factors, which are widely known to participate in gene expression regulation [9]. The MAF family consists of 7 members, which are grouped into "large" (c-Maf, MafA, MafB, and Nrl) and "small" (MafG, MafK, and MafF) Maf subfamily, based on their size [6]. Each member of the MAF family harbors a basic-leucine zipper (b-ZIP) domain involved in DNA binding and in dimer formation, either with themselves or with different b-ZIP transcription factors, in particular Nrf2 $[10,11]$. In addition to the b-ZIP domain, large Maf proteins also possess an acidic transcriptional 
activation domain (TAD) that, on the contrary, is absent in the small Mafs (sMafs). For this reason, the regulatory activity of small Mafs on gene transcription can be positive or negative, depending on their specific partner and on the promoter context. In general, homodimers of sMafs lacking TAD (i.e., MafG:MafK) repress gene transcription by binding to the Maf recognition element (MARE: TGCTGACT CAGCA) [6, 12]. The heterodimers with cap ' $n$ ' collar (CNC) proteins such as p45 NF-E2 [13] or with NF-E2related factors (Nrf1, Nrf2, and Nrf3) $[14,15]$ as well as with the Bach (BTB and CNC homology) factors (Bach1 and Bach2) [16], unlike homodimers, can either function as transcriptional activators or repressors [6]. Among these, as mentioned before, the Nrf2:sMaf heterodimers promote transcription and represent the most relevant means for adjusting a multitude of intracellular protein levels in response to oxidative/electrophilic stresses [5]. Under basal unstressed conditions, Nrf2 is polyubiquitinated and targeted to $26 \mathrm{~S}$ proteasome by the Kelch-like ECH-associated protein 1(Keap1)-Cul3 E3 ligase complex [17]. Stresses provoke dissociation of Keap1 through modification of specific cysteine residues [18]. Thus, Nrf2 can migrate into the nucleus, where in association with sMaf, in particular MAFG, binds to the promoters of target genes harboring ARE sequences to activate their transcription [8].

sMafs also interact with HIF- $1 \alpha$ to positively regulate hypoxic responses [19]. On the other hand, Bach1:sMaf heterodimers act as negative regulator of the HMOX-1 gene and its role is supported by genetic data [20]. In addition, Fang and colleagues recently demonstrated that high MAFG levels driven by BRAF (V600) recruit Bach1 as partner along with $\mathrm{CHD} 8$, a chromatin remodeling factor, and DNMT3B, a DNA methyltransferase, to trigger epigenetic silencing of genes frequently hypermethylated in melanoma and colorectal cancer [21].

In the last decades, microRNAs (miRs) have added a new layer of complexity to the regulatory mechanisms underlying gene expression $[22,23]$. miRs are small endogenous noncoding RNAs that downmodulate protein expression at posttranscriptional level. By interacting with specific regions, generally localized in the $3^{\prime}$-untranslated regions (UTRs) of transcripts, miRs mediate repression of mRNA translation or stability $[24,25]$. Given that each single miR could target multiple transcripts and often a miR regulates several targets of the same pathway, they supervise cellular signaling and networks involved in fundamental biological processes, including stress responses [26-28].

Numerous studies have shown that miR-128 is involved in different cellular processes such as differentiation, apoptosis, senescence, and metabolism [29-32]. Here, we investigated its role in the stress/antioxidant networks regulated by the heterodimeric complex Nrf2:MAFG. We demonstrated that miR-128 by targeting MAFG influences stress responses mediated by ARE-dependent genes.

\section{Materials and Methods}

2.1. Cell Cultures and Reagents. Human embryonic kidney HEK293 cells and mouse myogenic C2C12 cells were obtained from American Type Culture Collection (ATCC, Manassas, VA, USA). Cells were cultured in Dulbecco's modified Eagle's medium (DMEM) (Thermo Fisher SCIENTIFIC, Italia, Monza, Italy) supplemented with fetal bovine serum (FBS) (Invitrogen) at 10\% for HEK293 and 20\% for C2C12 cells. To induce differentiation of $\mathrm{C} 2 \mathrm{C} 12$ cells, culture medium was replaced to near-confluent cultures (about 90\%) with DMEM containing 2\% horse serum (Thermo Fisher SCIENTIFIC).

Hypoxia $\left(2 \% \mathrm{O}_{2}\right)$ for $4 \mathrm{~h}$ was induced when $\mathrm{C} 2 \mathrm{C} 12$ cells were at $85 \%$ confluence. After being washed three times with phosphate-buffered saline (PBS), cultures were transferred to a $37^{\circ} \mathrm{C}$ incubator within a hypoxic chamber $\left(93 \% \mathrm{~N}_{2}, 5 \%\right.$ $\mathrm{CO}_{2}, 2 \% \mathrm{O}_{2}$ ), and culture medium was replaced with a saline buffer that was prebubbled for five minutes with the same gas mix, to provide an average $\mathrm{O}_{2}$ pressure $(14.7 \mathrm{mmHg}$ ) equivalent to that in the ambient air of the chamber.

Treatments with diethylmaleate (DEM) (Sigma-Aldrich, Milan, Italy) were performed for $2 \mathrm{~h}$ (HEK293) or $4 \mathrm{~h}$ (C2C12) using a final concentration of $200 \mu \mathrm{M}$ in complete culture medium.

2.2. Plasmid Constructs and Transfections. The plasmid expressing miR-128 (pCMV-miR-128) was obtained by cloning the human ARPP21 intronic region of $284 \mathrm{bp}$ encompassing the pre-miR-128-2 sequence with $100 \mathrm{bp}$ upstream and downstream flanking sequences into pRcCMVneo vector. This fragment was prepared from human IMR90 DNA by PCR using the following oligonucleotides: 5 '-ACgTA AAAgCTTAAgAAggCTATTgACAATCCAg- $3^{\prime}$ and $5^{\prime}-\mathrm{CgA}$ CATATCTAgATTTggTCAgCAggAATgaCAC harboring HindIII and XbaI restriction sites, respectively. The identity of the cloned region was established by digestion and confirmed by sequencing. The expression of mature miR-128 was evaluated by Northern blot (Supplementary Materials available online at https://doi.org/10.1155/2017/9308310) in HEK293 cells and miR-128 levels measured by reverse transcription-quantitative real-time PCR (RT-qPCR).

The luciferase constructs were produced by cloning the $3^{\prime}$ UTR regions with the miR-128 site/s of candidate genes into the pGL3-control vector, downstream the Firefly luciferase gene (Promega, Madison, WI, USA). The $3^{\prime}$ UTR fragment of MAFG was amplified by PCR using human IMR90 DNA with primer pairs containing $\mathrm{XbaI}$ sites: $5^{\prime}$-ATCATTCTA GAGGATCCATGCAGGCATGCTGGCTCC-3' and $\overline{5^{\prime}-\mathrm{AT}}$ CATTCTAGATTCAAGCTACTATCAGACAATGT $-3^{\prime}$. The $3^{\prime} \mathrm{UTR}$ of $\mathrm{Nrf} 2$ was obtained from the full length cDNA (I.M.A.G.E.: 4548874) by using the following primers with Xba sites: $5^{\prime}$-ACGTACTCAGATTTAGGAGGATTTGAC CT- $3^{\prime}$ and $5^{\prime}$-ACGTACTCAGATAACAGTCATAATAAT CCTTTATTA- $3^{\prime}$. The orientation of the cloned fragments was established by digestion and confirmed by sequencing. The pGL3 constructs with the reverse orientation were used as negative controls. All the plasmids bearing the 3 UTR fragments were transfected in HEK293 cells with Lipofectamine 2000 Reagent (Thermo Fisher SCIENTIFIC) in 24-well plates (40.000 cells/well), according to the manufacturer's instructions, and cotransfected with synthetic pre-miR-128 (ID: 
PM1176) or pre-miR-negative control number 1 (100 nM) (Thermo Fisher SCIENTIFIC) as well as with pCMV-miR128 or pCMVneo (ratio 1:5). Transfections of cells with synthetic pre-miR-128 or pre-miR negative control were performed as previously described [33].

The plasmid expressing the FLAG-tagged Nrf2 and the plasmids GSTA1-LUC, $\mathrm{x}$-CT-LUC, and NQO1-LUC bearing the promoters of the respective genes driving the expression of luciferase reporter gene have been described previously $[34,35]$.

2.3. Luciferase Reporter Assay. The pGL3-3'UTR constructs $(100 \mathrm{ng})$ were cotransfected with the Renilla luciferase reporter plasmid (20 $\mathrm{ng}$ ) as an internal control using Lipofectamine 2000 (Thermo Fisher SCIENTIFIC) in HEK293 cells in the presence of miR-128 or negative control. Luciferase activity was measured at $24 \mathrm{~h}$ after transfection using a dual luciferase reporter assay (Promega) according to the manufacturer's instructions and performed on a $20 / 20^{\mathrm{n}}$ Luminometer (Turner BioSystems, Sunnyvale, CA, USA). Relative luciferase activity was calculated by normalizing the Firefly luminescence to the Renilla luminescence and then calculated relative to the control. $\mathrm{C} 2 \mathrm{C} 12$ cells plated in 24-well plates (30.000 cells/well) were cotransfected with the GSTA1-LUC, $\mathrm{x}$-CT-LUC, and NQO1-LUC constructs (500 ng) and FLAG-Nrf2 or empty vector (60 ng) and simultaneously with miR-128 expressing plasmid or empty vector (500 ng) using Lipofectamine ${ }^{\circledR}$ LTX Reagent (Thermo Fisher SCIENTIFIC) according to the manufacturer's instructions. All the transfections also contained a Renilla luciferase construct ( $40 \mathrm{ng}$ ) for internal normalization. Cells were then harvested at $36 \mathrm{~h}$ after transfections, and luciferase activity was determined as described above.

2.4. Western Blotting. Total proteins were extracted from transfected cells/treated cells with a buffer containing $0.02 \mathrm{M}$ HEPES (pH 7.9), 0.4 M NaCl, 0.1\% NP-40, 10\% $v / v$ glycerol, $1 \mathrm{mM} \mathrm{NaF}, 1 \mathrm{mM} \mathrm{Na} \mathrm{VO}_{3}$, and a protease inhibitor cocktail (Sigma-Aldrich). Cytoplasmic and nuclear proteins were fractionated as previously described [34], and cytosolic/nuclear fractions were evaluated by using the antibody of UCHL3 [36]. Cellular protein extracts were loaded on SDS-PAGE, followed by blotting to PVDF membranes. After blocking in nonfat milk solution, membranes were probed with specific primary antibodies (indicated below) and then incubated with horseradish peroxidase-conjugated secondary antibodies for $1 \mathrm{~h}$. Protein bands were visualized using the ECL chemiluminescence system (Amersham, Buckinghamshire, UK).

Murine adductor muscles were homogenized using the program Protein_1 on a gentleMACS tissue Dissociator (Miltenyi Biotec) [37]. Briefly, tissues were lysated in a buffer containing $50 \mathrm{mM}$ Tris- $\mathrm{HCl}$ (pH 7.4), $150 \mathrm{mM} \mathrm{NaCl}, 1 \% \mathrm{NP}-$ 40, $1 \mathrm{mM}$ EDTA, $0.25 \%$ sodium deoxycholate, $10 \mathrm{mM} \mathrm{NaF}$, $10 \mu \mathrm{M} \mathrm{Na} \mathrm{VO}_{3}$, $1 \mathrm{mM}$ PMSF, and protease inhibitor cocktail $(10 \mathrm{~g} / \mathrm{mg}$ aprotinin, $10 \mathrm{~g} / \mathrm{ml}$ pepstatin, and $10 \mathrm{~g} / \mathrm{ml}$ leupeptin). Lysates were centrifuged at $14,000 \mathrm{rpm}$ for $15 \mathrm{~min}$, and protein concentrations were measured using Bio-Rad assay kit (Bio-Rad) and immunoblotting was performed as described [38].

MAFG antibody was from GeneTex (GTX114541); HIF- $1 \alpha$ antibody was from Novus Biologicals (NB100-105). Antibody of FLAG M2 was from Sigma-Aldrich. Antibodies of Nrf2 (number SC-13032), BMI-1 (number SC-390443), HMOX-1 (number SC-136960), tubulin (number SC-8035), UCHL3 (number SC-100340), and vinculin (number SC7649) were from Santa Cruz Biotechnology Inc., Santa Cruz, CA, USA.

2.5. Reverse Transcription-Quantitative Real-Time PCR (RT$q P C R)$. Total RNA was extracted using TRIzol reagent (Thermo Fisher SCIENTIFIC), and cDNA was synthesized from one $\mu \mathrm{g}$ of RNA using random primers and iScript cDNA synthesis kit (Bio-Rad Laboratories, Hercules, CA, USA). mRNA levels were quantified using IQ SYBR green supermix (Bio-Rad Laboratories) on the CFX96 real-time system instrument (Bio-Rad). Specific primers located in different exons of the same gene were designed to detect relative mRNA levels. The housekeeping $\beta-2$ microglobulin or c-ABL genes were used for internal normalization. All the PCR reactions were performed in triplicate, and PCR products were also visualized on agarose gels after ethidium bromide staining. The oligonucleotide sequences are reported in Supplemental Table S2. Relative fold variations were calculated using the $2^{-\Delta \Delta C t}$ method by the formula: $2^{-(\text {sample } \Delta \mathrm{Ct} \text {-control } \Delta \mathrm{Ct})}$, where $\Delta \mathrm{Ct}$ is the difference between the amplification fluorescent thresholds of the gene of interest and the internal reference gene/s used for normalization [39].

To detect the levels of mature miR-128, total RNA was prepared with TRIzol reagent (Thermo Fisher SCIENTIFIC) and miR amounts were evaluated by using the TaqMan miRNA assay kit (Thermo Fisher SCIENTIFIC). For normalization of RNA levels, the amounts of small nucleolar RNA RNU6 (Thermo Fisher SCIENTIFIC) were measured [39].

2.6. Animal Studies. All experiments involving animals were conforming to the Guide for the Care and Use of Laboratory Animals published by the US National Institutes of Health (NIH Publication 8th edition, update 2011) and were approved by the animal welfare regulation of the University of Naples Federico II, Italy. Wild-type C57BL/6 male mice (age 8 to 9 weeks) were included in the study and maintained under identical conditions of temperature $\left(21 \pm 1^{\circ} \mathrm{C}\right)$, humidity $(60 \pm 5 \%)$, and light/dark cycle and had free access to normal mouse chow.

2.7. Peripheral Ischemia Procedure. Mice were anesthetized with an intraperitoneal injection of $1 \mathrm{ml} / \mathrm{kg}(50 \mathrm{mg} / \mathrm{kg})$ of a mixture of $50 \%$ tiletamine and $50 \%$ zolazepam $(50 \mathrm{mg} / \mathrm{ml}$ tiletamine and $50 \mathrm{mg} / \mathrm{ml}$ di zolazepam, Zoletil 100) plus xylazine $5 \mathrm{mg} / \mathrm{kg}$ (Sigma-Aldrich). The adequacy of anaesthesia was confirmed by the absence of reflex response to foot squeeze. Hindlimb ischemia was induced as previously described (PI, $n=7$ ) [40]. Briefly, the proximal and distal portions of the left femoral artery were ligated and arteriectomy was performed between these two sites. Mice were laid 
on a heating pad $\left(37^{\circ} \mathrm{C}\right)$ under anaesthesia, and their blood flow was measured by a laser Doppler perfusion imager (Perimed, Periscan, USA) in the ischemic and nonischemic limbs before and 0 and 4 hours after surgery, to confirm vascularization impairment after the procedure. SHAMoperated animals underwent the same procedure without femoral artery ligation (SHAM, $n=4)$. All SHAMoperated controls and all mice subjected to peripheral ischemia (PI) survived.

2.8. Statistical Analysis. For the statistical analyses, the Student's $t$-test was used; data were considered significant at a value of $p<0.05$.

\section{Results}

3.1. MAFG Is a Novel Direct Target of $m i R-128$. A previous study of Venkataraman et al. reports that miR-128 promotes intracellular ROS increases [31]. Given that Nrf2 signaling is the main driver of the transcriptional program inducing the expression of a wide range of antioxidant genes [3-5], we hypothesized that miR-128 would affect this regulatory pathway. Thus, we used in silico prediction programs to search for putative targets of miR-128 among the components of Nrf2 pathway. TargetScan, miRanda, and RNAhybrid algorithms predicted conserved seed region/s of miR-128 in the 3'UTRs of Nrf2 and MAFG as well as on the Nrf2-regulated gene $\mathrm{x}$-CT (Supplemental Table S1). Since Nrf2 and MAFG are transcription factors and both implicated in the activation of ARE-dependent transcription [8], we performed further analyses on these genes. To assess whether Nrf2 and MAFG are direct targets of miR-128, the entire 3 'UTR of Nrf2 and a region encompassing the mir-128 seed sequence of MAFG (250 bp) were cloned into the pGL3-vector downstream of the luciferase gene. Moreover, to validate specificity of miR-128 interaction with 3 'UTRs, mutant reporter constructs (mut) harboring the same regions cloned in the opposite orientation were prepared. Figure 1(a) shows that ectopic expression of synthetic pre-miR-128 slightly decreases the luciferase activity of the construct harboring the $3^{\prime} \mathrm{UTR}$ region (wt) of Nrf2 in HEK293 cells. In the same conditions, miR-128 overexpression however significantly $(p<0.05)$ reduced the activity of the reporter construct bearing the wild-type $3^{\prime}$ UTR (wt) of MAFG compared to miR-SCRtransfected cells (Figure 1(b)). Conversely, mutant MAFG reporter construct (mut) did not respond to miR-128 increases (Figure 1(b)).

To set up a more physiological miR-128 hyperexpression, we also prepared a construct (pCMV-miR-128) that drives miR-128 overexpression from the ARPP21 genomic region (see Materials and Methods). We confirmed that ectopic expression of miR-128 through pCMV-miR-128 (of about 100 folds at $24 \mathrm{~h}$ posttransfection, Supplemental Figure S1) in HEK293 cells indeed reduced the luciferase activity of the MAFG-3'UTR reporter construct while there was no effect on the Nrf2-3'UTR construct, very similar to the results obtained with synthetic pre-miR-128 (data not shown).

To validate the above results on the expression of endogenous MAFG protein, we next performed Western blotting analysis on extracts from HEK293 cells transfected for $24 \mathrm{~h}$ either with synthetic pre-miR-128 or with pCMV-miR-128. Figure 1(c) shows that endogenous protein levels of MAFG are significantly reduced in condition of miR-128 overexpression, compared to the negative control-transfected cells. In the same conditions, the downregulation of BMI-1 protein levels, a known miR-128 target, is also detected (Figure 1(c)).

We also observed that miR-128 hyperexpression correlates with MAFG downregulation (Supplemental Figure S2) in physiological/pathological conditions, like differentiation of mouse C2C12 myoblasts [41, 42] or atrophic stimulation of differentiated C2C12 [43]. Taken together, the above reported results suggest that miR-128 represses the expression of the MAFG protein.

3.2. miR-128 Influences the Basal Expression of Some MAFG-Regulated Genes. Following the observation that MAFG is negatively regulated by $\mathrm{miR}-128$, we aimed to test the effects of miR-128 on MAFG-dependent transcriptional activity. To this end, we examined the mRNA levels of various MAFG-regulated genes [15, 44] such as AKR1D1, ALDH3, CCDC53, HMOX-1, Nrf2, PCBD2, and UCHL1 upon miR-128 hyperexpression (Figure 2(a)). The results show that in HEK293 cells, the basal expression of AKR1D1, ALDH3, and PCBD2 genes was reduced in conditions of miR-128 overexpression. These results further support a role of miR-128 in the regulation of a set of MAFGdependent genes.

MAFG is a known basic leucine zipper (bZIP) protein that was shown to interact with other transcription factors, in particular with Nrf2 to activate the expression of numerous genes [7, 15]. Furthermore, MAFG could enhance nuclear retention of Nrf2 [45]. Therefore, we next determined whether the Nrf2 levels are influenced by miR-128 overexpression. Nrf2 is present at very low levels in the cells; thus to stabilize its levels, we exposed wild-type HEK293 and transfected HEK293 cells to low concentration of diethylmaleate (DEM), a glutathione depleting agent [46], for $2 \mathrm{~h}$ before harvesting. Western blotting analysis was used to detect the endogenous Nrf2 protein levels after exposure to DEM (Figure 2(b)). In HEK293 control cells, Nrf2 was found substantially increased in the cytosol and also weakly enhanced in the nucleus by DEM exposure. In the same conditions of treatments, cells transfected with miR-128 did not result in reduced accumulation/translocation of Nrf2 compared to cells transfected with empty vector. These results are also in line with the data obtained by 3'UTR luciferase assays that demonstrated that miR-128 is not involved in Nrf2 posttranscriptional regulation.

Since miR-128 has site/s in the 3'UTR of MAFG conserved among mammals (Supplemental Table 1) and since targets of miRNA are cell-type specific, we also examined the effect of miR-128 on the expression of endogenous mouse MAFG using the mouse myogenic C2C12 cell line. The results showed that ectopic expression of miR-128 also promotes downregulation of MAFG protein in the mouse context (Figure 2(c)). In the same conditions, as expected, BMI-1 protein levels were downregulated. In parallel, we evaluated whether the changes observed for MAFG- 


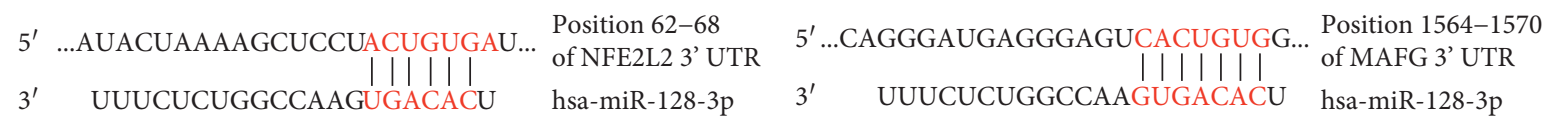

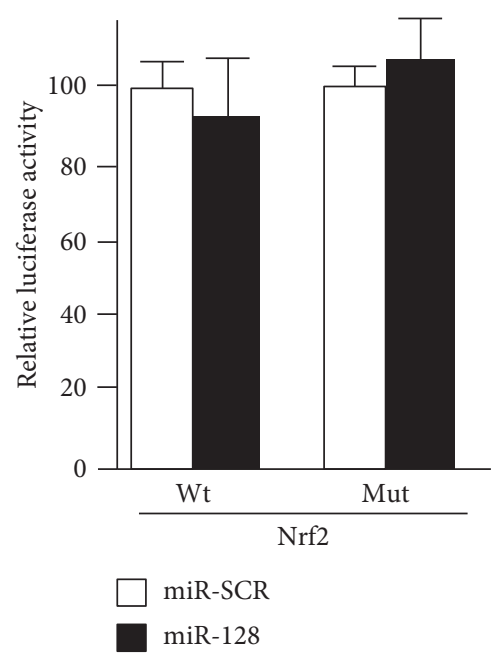

(a)

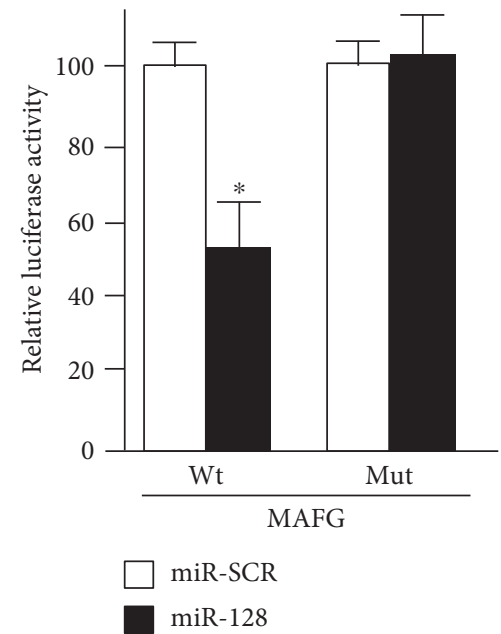

(b)

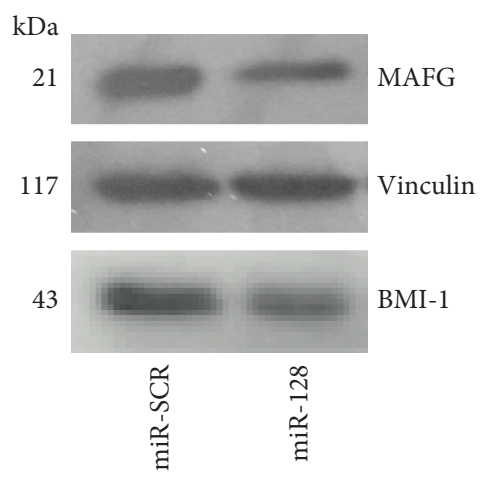

(c)

FIGURE 1: Validation of miR-128 targets by luciferase assay in HEK293 cells. (a) HEK293 cells were transiently transfected with the luciferase construct bearing wild-type (wt) or inverted (mut) 3'UTR fragment holding putative miR-128 target sites of human Nrf2. The alignment of miR-128 with Nrf2-3'UTR is indicated at the top. Cells were simultaneously transfected with either $100 \mathrm{nM}$ of pre-miR-128 (miR-128) or pre-miR negative control (miR-SCR). The pRLSV40 encoding Renilla luciferase plasmid was used as an internal control. Dual luciferase assays were performed as described in the Materials and Methods. Transfections were performed in triplicate, and the ratio ( \pm SD) Firefly/ Renilla luciferase activity from three independent experiments were averaged and expressed as percentage of the respective transfections performed with miR-SCR control. ${ }^{*} p<0.05$. (b) Luciferase constructs containing wild-type (wt) or mutated (mut) 3 'UTR of MAFG with predicted miR-128 site (indicated on the top) were cotransfected with either $100 \mathrm{nM}$ of pre-miR-128 (miR-128) or pre-miR negative control (miR-SCR) in HEK293 cells and luciferase activity quantified as described in (a). ${ }^{*} p<0.05$. (c) Western blotting analysis of MAFG and BMI-1 levels on total proteins from HEK293 cells transfected for $24 \mathrm{~h}$ with either $100 \mathrm{nM}$ of pre-miR-128 (miR-128) or pre-miR negative control (miR-SCR); vinculin was used as a loading control.

dependent genes in HEK293 are also present in mouse C2C12 after miR-128 overexpression. As shown in Figure 2(d), the basal mRNA levels of AKR1D1, ALDH3, and UCHL1 genes were reduced in conditions of miR-128 hyperexpression. These results support a role of miR-128 in the regulation of MAFG protein levels and of MAFGdependent genes also in mouse contexts.

3.3. Ectopic Expression of miR-128 Influences the Expression of an Array of ARE-Dependent Genes. MAFG as bZIP protein interacts with Nrf2 to facilitate its binding to the ARE consensus within the regulatory regions of numerous cytoprotective genes. To determine whether MAFG downregulation through $\mathrm{miR}-128$ also affects the basal transcription of an array of genes that are typically regulated by the Nrf2:MAFG heterodimer $[8,11]$, we examined the mRNA levels of representative genes such as GCLC, GSTA-1, NQO1, $\mathrm{x}-\mathrm{CT}$, and SQSTM1 in C2C12 cells overexpressing miR-128. The mRNA levels of Nrf2 and p $21^{\mathrm{WAF} 1}$ were used as controls, since it has been demonstrated that these genes are not dependent on MAFG regulation [8]. As shown in Figure 3(a), the mRNA levels of GCLC, NQO1, and SQSTM1 were significantly $(p<0.05)$ downregulated in C2C12 cells, whereas other Nrf2 target genes such as $\mathrm{x}$-CT and GSTA-1 


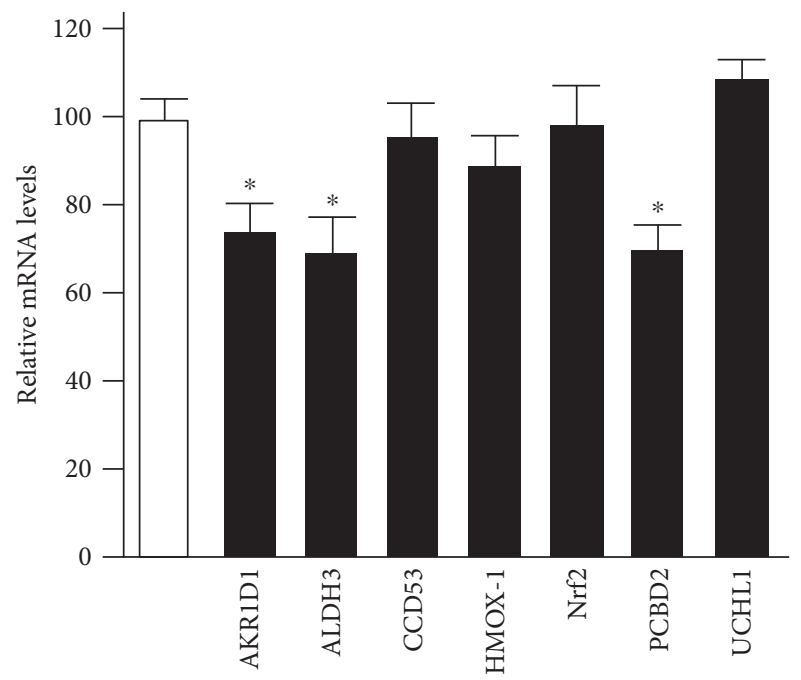

CMV-neo

CMV-miR-128

(a)

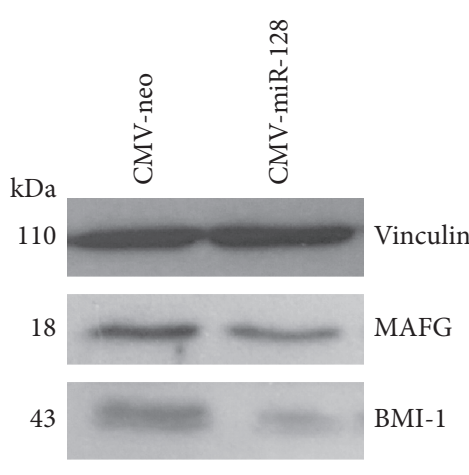

(c)

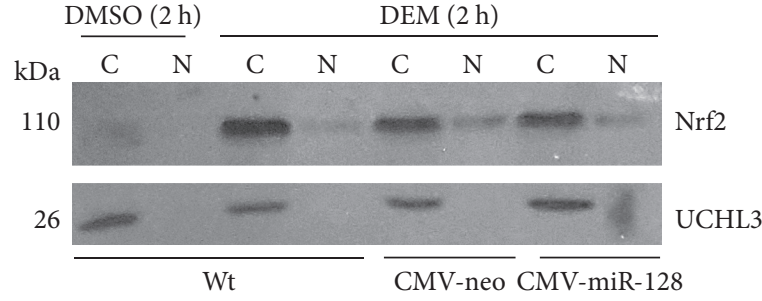

(b)

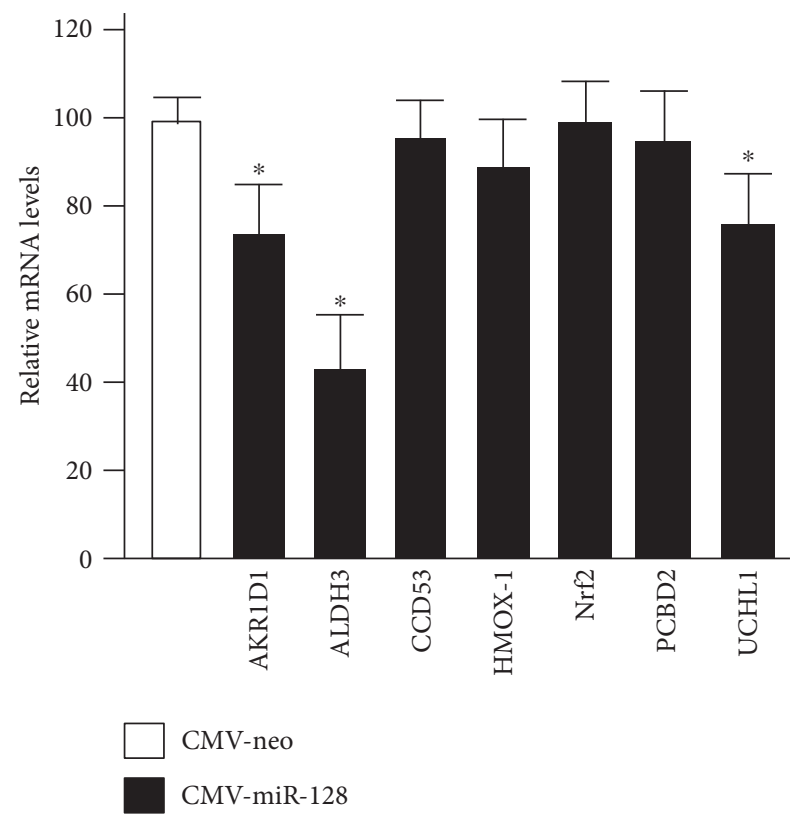

(d)

FIGURE 2: miR-128 downregulates the expression of MAFG and MAFG-regulated genes in human and mouse contexts. (a) HEK293 cells were transfected with either CMV-miR-128 construct or negative control vector (CMV-neo). After $24 \mathrm{~h}$, total RNAs were isolated and the relative mRNA levels of the indicated genes were calculated through quantitative real-time PCR by comparing the levels associated to miR-128 expressing- versus CMV-neo control cells, after normalization with c-ABL. Data were reported as relative to the vector control, which was set equal to 100. Each column in the panel represents the mean \pm SD of 3 independent experiments. ${ }^{*} p<0.05$. (b) HEK293 cells were transfected with either pre-CMV-miR-128 or negative control (CMV-neo). After $22 \mathrm{~h}$, cells were treated for $2 \mathrm{~h}$ with $200 \mu \mathrm{M}$ DEM. Untransfected HEK293 cells were used as positive control for DEM treatment. Nrf2 protein levels were determined by Western blotting on cytosolic [C] and nuclear [N] extracts. UCHL3 was used as a control of extract preparations. (c) CMV-neo or CMV-miR-128 were transiently transfected into $\mathrm{C} 2 \mathrm{C} 12$ cells for $30 \mathrm{~h}$, and Western blotting analysis of MAFG and BMI-1 was performed on total protein extracts; vinculin was used as a loading control. (d) mRNA levels of AKR1D1, ALDH3, CCDC53, HMOX-1, Nrf2, PCBD2, and UCHL1 genes were analyzed as described in (a) at $30 \mathrm{~h}$ after transfection in $\mathrm{C} 2 \mathrm{C} 12$ cells. Each column in the panel represents the mean \pm SD of at least 3 independent experiments. ${ }^{*} p<0.05$. 


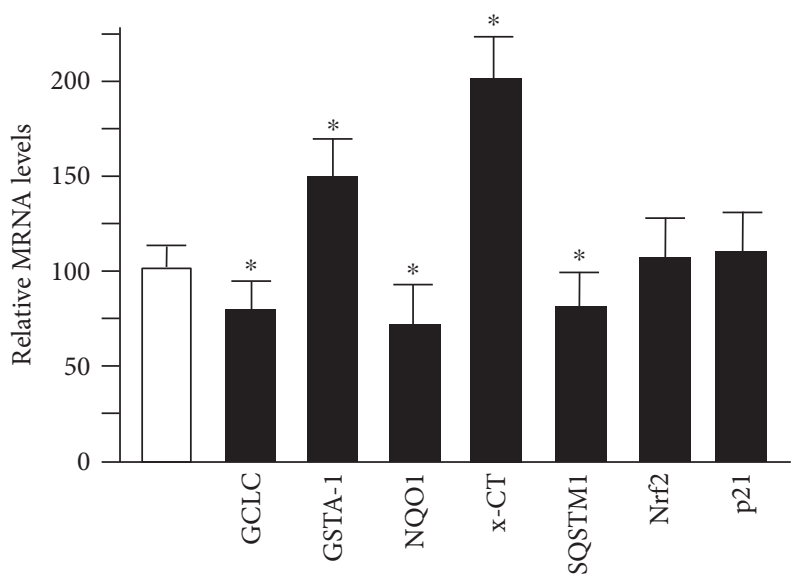

$\square$ CMV-neo
CMV-miR-128
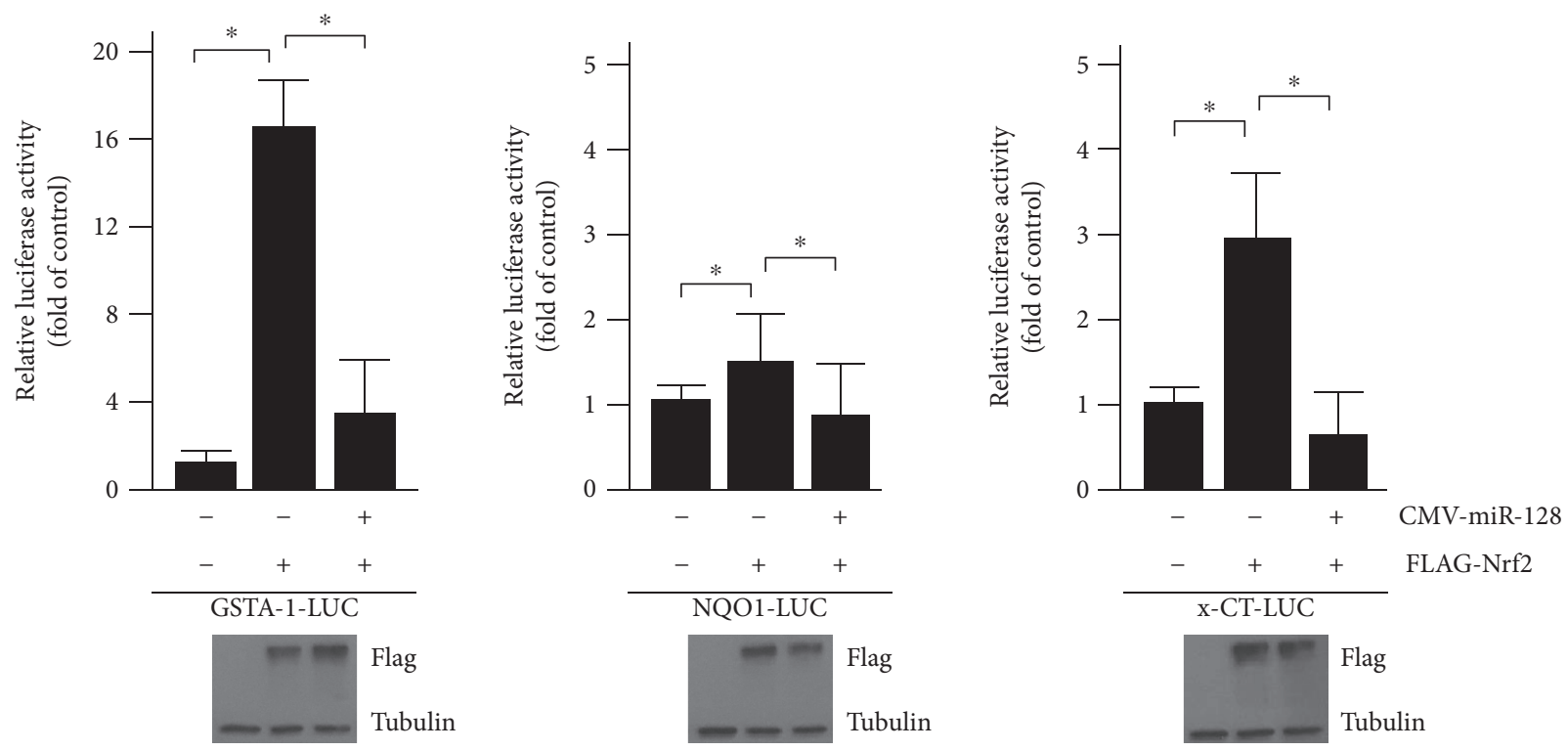

(b)

FIGURE 3: miR-128 provokes modifications of ARE-dependent genes at basal levels and impairs Nrf2 activity. (a) Analysis of GCLC, GSTA-1, NQO1, $x-C T$, SQSTM1, Nrf2, and p21WAF1 expression in C2C12 cells by RT-qPCR after miR-128 overexpression. mRNA levels in miR-128-transfected cells are expressed as percentage of the levels found in cells transfected with CMV-neo, after normalization with the c-ABL. Each column represents the mean \pm SD of 3 independent experiments of transfections. ${ }^{*} p<0.05$. (b) The promoter regions of GSTA-1 (GSTA1-LUC) or NQO1 (NQO1-LUC) or x-CT (x-CT-LUC) containing ARE motifs cloned into PGL3 basic vector were transiently cotransfected with pRLSV40 encoding Renilla luciferase, in the presence or absence of miR-128 expressing vector. They were also cotransfected with FLAG-Nrf2 or with equivalent amount of empty plasmid, as indicated. $30 \mathrm{~h}$ after transfections, the Firefly/Renilla luciferase activities were assessed and the values were reported as relative to the luciferase activities obtained with the respective LUC construct, which were set equal to 1 . The results are representative of three independent experiments, each performed in triplicate. ${ }^{*} p<0.05$. Representative Western blotting of FLAG-Nrf2 overexpression is also presented. Tubulin protein levels were used as loading controls.

were upregulated. These findings indicate that protein reduction of MAFG following miR-128 overexpression influences the basal expression of some ARE-dependent genes in $\mathrm{C} 2 \mathrm{C} 12$ cells. The above results also suggest that lowered basal expression of GCLC and NQO1 after miR-128 overexpression could explain the ROS increase observed by other authors [31].
Increased levels of Nrf2:MAFG heterodimer activate the transcription of a wide variety of genes containing ARE sequences in their promoters [8]. Therefore, we analyzed the ability of miR-128 to influence the Nrf2 activity on ARE regulatory regions of selected genes. To this aim, we used luciferase reporter constructs, namely, GSTA1-LUC, NQO1-LUC, and $\mathrm{x}$-CT-LUC $[34,35]$, that in conditions of $\mathrm{Nrf} 2$ 

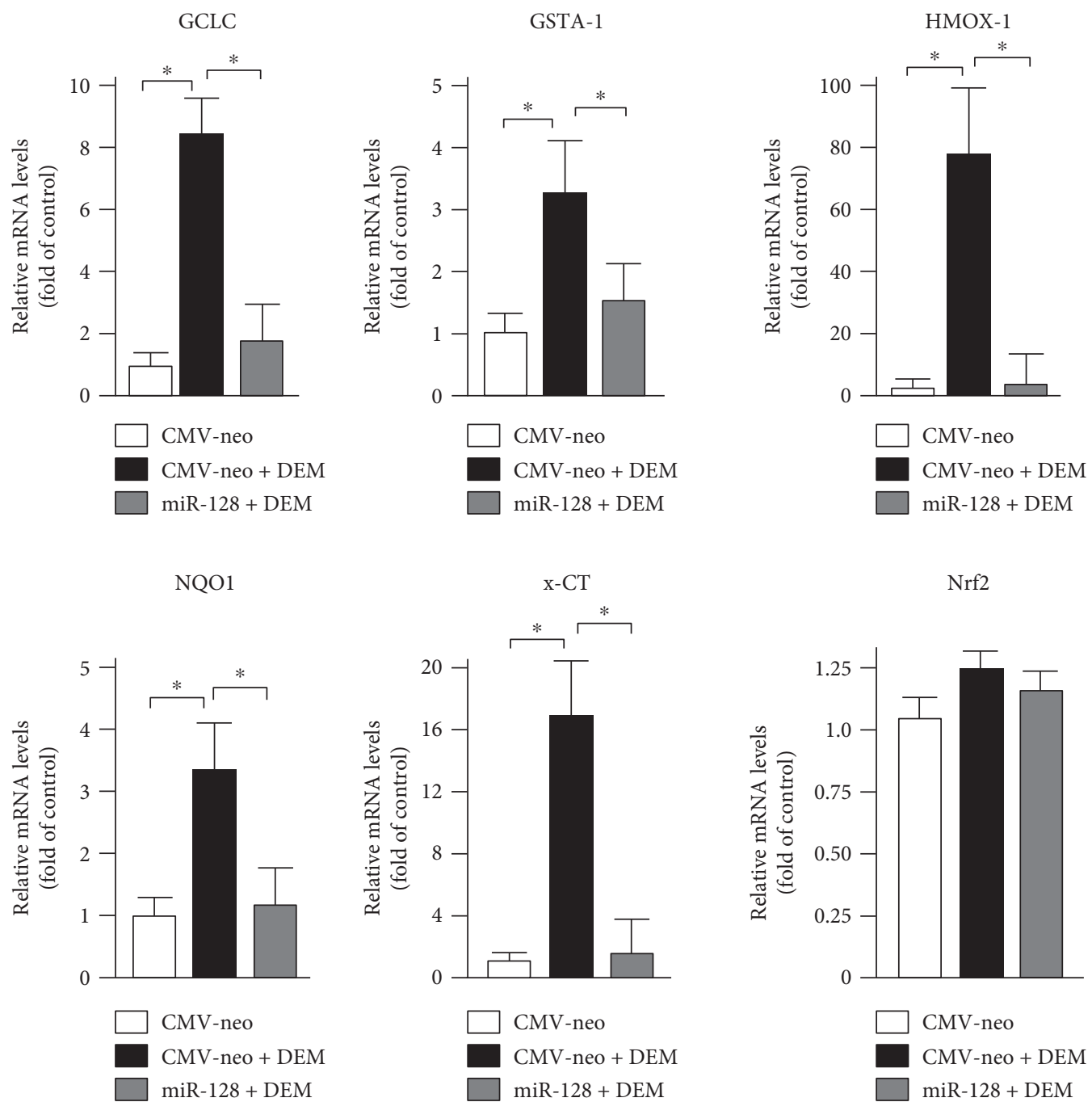

FIGURE 4: miR-128 interferes with the DEM-mediated induction of ARE-dependent genes. C2C12 cells were transiently transfected with miR-128 expressing construct or with an equivalent amount of empty plasmid. $36 \mathrm{~h}$ after transfections, the cells were treated for $3 \mathrm{~h}$ with $200 \mu \mathrm{M}$ DEM and then harvested. The mRNA levels of GCLC, GSTA-1, HMOX-1, NQO1, x-CT, and Nrf2 genes were determined by RT-qPCR on total RNA after normalization with the c-ABL mRNA level. Data are expressed as relative to the values obtained upon transfection with CMV-neo control in the absence of DEM, which were set equal to 1. Each column in the panels represents the mean \pm SD of 3 independent experiments. ${ }^{*} p<0.05$.

overexpression strongly induce luciferase activity [34, 35]. Thus, the above LUC constructs were cotransfected in C2C12 cells along with a construct expressing FLAGNrf2 or with empty vector, in the absence or presence of miR-128. As shown in Figure 3(b), the luciferase activity driven by the regulatory regions of $\mathrm{x}-\mathrm{CT}$ and GSTA-1 genes is highly induced after ectopic expression of Nrf2, compared with the control vector, and these effects are completely abolished by the overexpression of miR-128. Even though the Nrf2-mediated activation of NQO1-LUC was less prominent, miR-128 significantly weakened this induction (Figure 3(b)). The expression of ectopic Nrf2 was validated by Western blotting analysis with anti-FLAG antibody.

These results suggested that miR-128 counteracts the Nrf2-mediated induction of ARE-dependent promoters through MAFG downmodulation. The differences observed between the ARE-responsive regions (Figure 3(b)) and the mRNA levels of endogenous GSTA-1 and x-CT (Figure 3(a)) genes could be due to the contributions of additional regulatory regions/transcription factors in the chromatin contexts.

3.4. miR-128 Interferes with the DEM-Mediated Induction of ARE-Dependent Genes. The effects described above led us to investigate whether a reduced expression of endogenous MAFG through miR-128 could result in dysregulation of endogenous stress responses. We selected a group of genes that are responsive to oxidative stress induced by DEM, a well-known inducer of ROS accumulation, by taking advantage of a published list of genes that are responsive to this agent in mouse context [8]. C2C12 cells were transfected with miR-128 or control vectors for $36 \mathrm{~h}$ and then exposed to DEM. RT-qPCR analysis was used to determine transcript levels of a small set of ARE-dependent genes, 
namely, GCLC, GSTA-1, HMOX-1, NQO1, and x-CT. As shown in Figure 4, upon DEM treatments, the endogenous mRNA levels of these genes are strongly induced in $\mathrm{C} 2 \mathrm{C} 12$ cells transfected with CMV-neo. In the same conditions of treatments, miR-128 overexpression significantly decreased the DEM-mediated induction of the above genes, when compared to control-transfected cells. In conclusion, miR128 by downregulating MAFG antagonizes the expression of ARE-dependent genes. The mRNA levels of Nrf2 transcripts, used as control gene, were unaffected [8].

3.5. miR-128 Downmodulation Regulates MAFG Increase in Hypoxic Condition Both In Vitro and In Vivo. Given that MAFG regulates hypoxic responses by interacting with HIF-1 $\alpha$ [19], we examined the involvement of miR-128MAFG axis in C2C12 cell responses to hypoxia. First, we estimated the endogenous levels of miR-128 after exposure to hypoxia $\left(2 \% \mathrm{O}_{2}\right)$ for $4 \mathrm{~h}$. RT-qPCR analysis for mature miR-128 amounts showed significant $(p<0.05)$ decreased levels of miR-128 after hypoxia in C2C12 (Figure 5(a)). To test whether the observed miR-128 changes influence, also MAFG protein levels, we performed Western blotting analysis of MAFG and HMOX-1, which is a common target of HIF- $1 \alpha$ and Nrf2 transcription factors. The results show that the protein levels of HMOX-1, as expected, are induced following treatment of $\mathrm{C} 2 \mathrm{C} 12$ cells within $4 \mathrm{~h}$ of low $\mathrm{O}_{2}$. Importantly, at the same time, we observed an increase of MAFG protein, which correlates with an enhanced HIF- $1 \alpha$ expression (Figure 5(b)).

Since hypoxia induces ROS production, we also measured the mRNA levels of selected ARE-regulated genes such as HMOX-1 and X-CT. In this condition, the mRNA levels of these genes are strongly induced after exposure of C2C12 cells to hypoxia (Figure 5(c)). These results indicate that miR-128 downregulation could be important for adjusting the MAFG levels necessary for both HIF- $1 \alpha$ transactivation and AREdependent gene induction in hypoxic conditions in vitro.

Finally, we analyzed the role of miR-128-MAFG axis in the ischemic process in vivo. We induced peripheral ischemia (PI) by femoral artery ligation for $4 \mathrm{~h}$ in C57BL/6J mice $(n=4)$. Sham-operated animals $(n=3)$ underwent the same surgical procedures without ligation of femoral artery. Hindlimb blood flow was analyzed by ecolordoppler preand $4 \mathrm{~h}$ after surgical procedures (Figure 5(d)). Western blotting experiments show that MAFG protein levels are significantly induced in the hindlimb muscle lysates (Figure 5(e)). We also investigated whether the upregulated levels of MAFG are inversely correlated to miR-128 amounts. RT-qPCR analysis for mature miR-128 levels show a significant decrease of miR-128 levels (Figure 5(f)).

Overall, these results indicate that miR-128-MAFG axis is an important contributor for responses to hypoxia both in vitro and in vivo.

\section{Discussion}

miRs play crucial roles in the regulation of gene expression. miRs are considered primarily "fine-tuning" regulators of protein activities, and thus they could balance the antioxidant responses. Recent studies indeed indicate that many miRs called redoximiRs can be either direct or indirect effectors of redox-related pathways [47]. In particular, several evidences demonstrate that, on one hand, Nrf2 itself can be a direct target of miRs and that, on the other hand, Nrf2 modulators, such as Keap1 and Bach1, can be regulated by miRs [5, 48-51]. The transcription factor Nrf2 participates in the adaptive responses to oxidative stress by enhancing transcription of many genes encoding antioxidant/detoxification enzymes, and impaired Nrf2 functions decrease tolerance to oxidative/chemical insults [5].

Herein, we identified a new function of miR-128 as redoximiR, which has a direct role in suppressing MAFG expression. Based on complementary screening for MAFGdependent genes as well as for ARE-dependent genes functioning through Nrf2:MAFG heterodimer or Nrf2-regulated genes, we demonstrate that miR-128 interferes with the induction of a group of selected genes, namely, GCLC, GSTA-1, HMOX-1, NQO1, and X-CT under oxidative stress. In fact, MAFG acts as positive partner of $\mathrm{Nrf} 2$ and its downmodulation by miR-128 consequently affects the Nrf2/ARE pathway.

Deregulated redox signaling and diminished antioxidant defenses represent a major cause of pathophysiological processes including cardiovascular and neurodegenerative diseases, cataracts, diabetes, and cancers, most of which are age related. Therefore, pharmacological/natural strategies addressed to an increase of Nrf2 activity could be beneficial for preventing oxidative stress-related diseases [52-55].

Hypoxia is known to produce ROS, suggesting a crosstalk between HIF- $1 \alpha$ and the Nrf2 pathways [56]. Since MAFG is related to both of these signalings, we also analyzed the involvement of miR-128 in hypoxic responses. Our results indicate that miR-128-MAFG axis significantly contribute in responses to hypoxia in $\mathrm{C} 2 \mathrm{C} 12$ cells and the decrease of miR-128 is parallel with MAFG induction, which consequently could affect MAFG-related genes such as HMOX-1 and $\mathrm{x}$-CT. Of note, the responses observed in vitro are also observed during the ischemic process in vivo.

Several studies demonstrate the involvement of miR-128 in different cellular processes such as differentiation, apoptosis, senescence, and metabolism [29-32]. Furthermore, miR128 is found downregulated under hypoxic conditions in human trophoblasts [57] and a recent study implicates a protective role in vivo of miR-128 inhibition during myocardial I/R injury [58]. We demonstrate that mir-128 is involved in the modulation of the antioxidant responses in vitro as well as in ischemic condition both in vitro and in vivo. Indeed, repression of miR-128 parallels with MAFG increases that consequently could affect MAFG-related genes such as HMOX-1 and $\mathrm{x}-\mathrm{CT}$. Our findings further suggest that downmodulation of miR-128 might contribute to the coordination of the adaptive hypoxic reprogramming that among many others involves genes that are dependent on MAFG, now considered a regulatory hub for numerous transcription factors $[8,15,19]$.

Ischemic cardiovascular diseases are a leading cause of morbidity and mortality in developed countries [59]. Even though the detrimental role of ROS generated during ischemia 


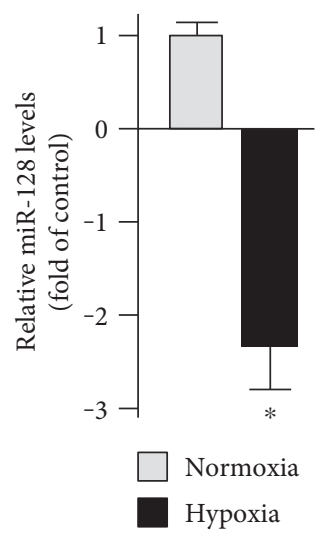

(a)

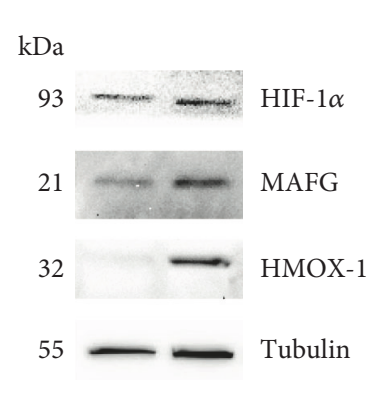

$\square$ Normoxia

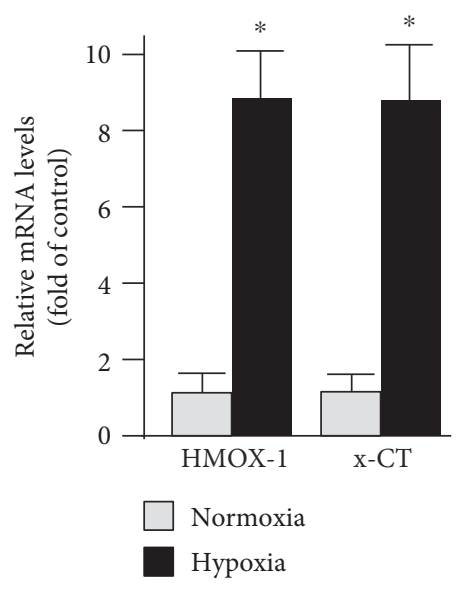

(c)

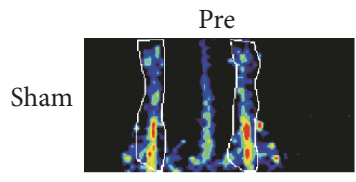

Post 4 hrs

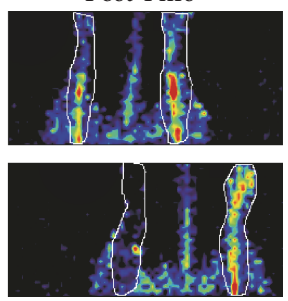

(d)
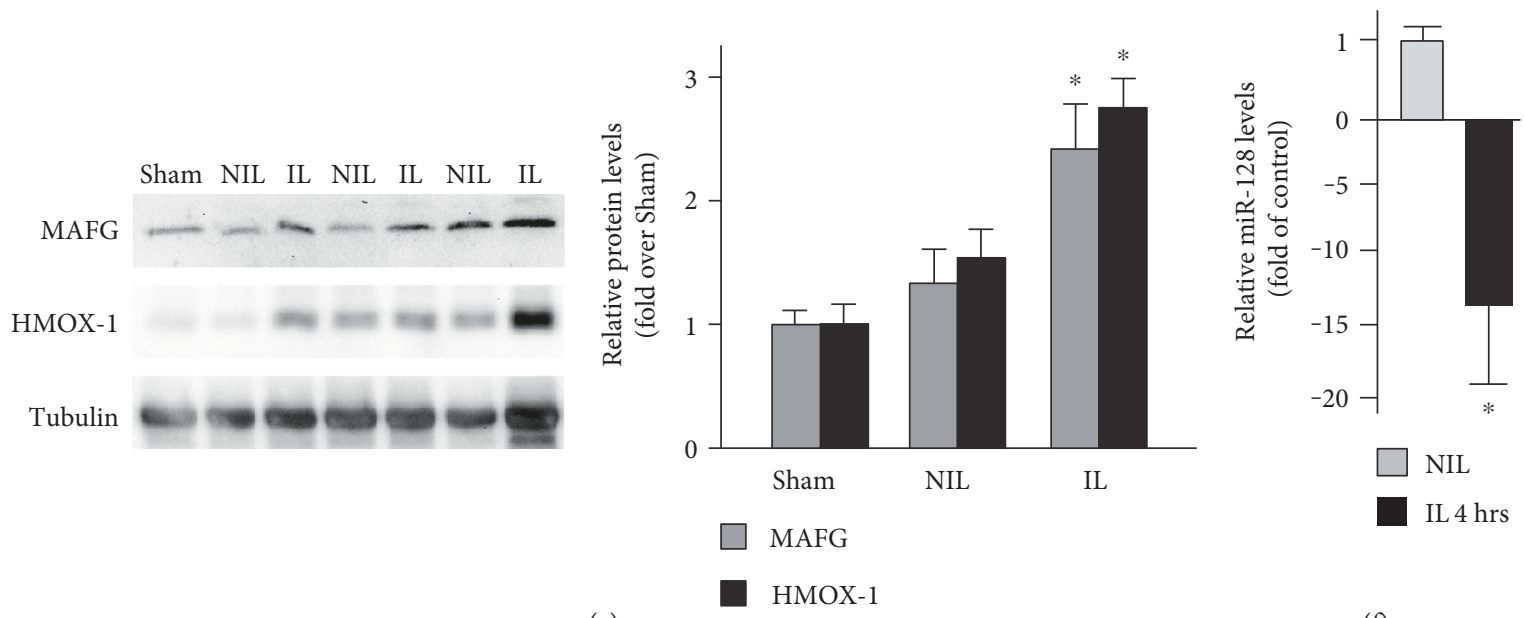

(e)

$(\mathrm{f})$

FIGURE 5: miR-128 downmodulation regulates MAFG increase in hypoxic condition both in vitro and in vivo. C2C12 cells were exposed to hypoxia ( $2 \% \mathrm{O} 2)$ for $4 \mathrm{~h}$, and miR-128 levels (a) were determined by RT-qPCR on total RNAs after normalization with the small RNU6. Data are expressed as relative to the values obtained in untreated control which were set equal to 1 . Each column in the panels represents the mean \pm SD of 3 independent experiments. ${ }^{*} p<0.05$. Western blotting analysis (b) of HIF- $1 \alpha$, MAFG, and HMOX-1 was performed on total extracts from the same cells, as described in Materials and Methods. Tubulin was used as a loading control. mRNA levels of HMOX-1 and $\mathrm{x}$-CT genes (c) were determined by RT-qPCR on total RNAs, and relative changes were calculated by comparing treated cells versus control, after normalization with c-ABL. Data were reported as relative to the values obtained in normoxia, which were set equal to 1 . Each column in the panel represents the mean \pm SD of 3 independent experiments. ${ }^{*} p<0.05$. Representative laser Doppler analysis (d) of blood flow before and 4 hours after (post $4 \mathrm{~h}$ ) hindlimb ischemia procedure ( $n=3-4$ mice/group). Representative Western blot analysis and densitometric analysis (e) of MAFG and HMOX-1 protein levels performed on hindlimb muscle lysates from littermates after sham procedure (sham) or not-ischemic limb (NIL) and ischemic limb (IL) $4 \mathrm{~h}$ after femoral artieriectomy. Tubulin protein levels were used as loading controls $\left({ }^{*} p<0.05\right.$ versus sham; $n=3-4$ hindlimb/group). miR-128 levels (f) were determined by RT-qPCR on total RNAs after normalization with the small RNU6. The values obtained in not-ischemic limb (NIL) were set equal to 1 . The data are expressed as the mean \pm standard error and are representative of 3 independent experiments. ${ }^{*} p<0.05$. 
is well demonstrated, an effective antioxidant therapy is still far to be determined. Many studies indeed indicate that Nrf2 protects against cardiovascular diseases albeit reductive conditions also generate $\operatorname{ROS}$ (reviewed in [60, 61]). In this context, our work highlights a novel possibility to manage the activities of the Nrf2 signal by miR-128, alone or in combination with other pharmacologic strategies, that in future studies can be considered.

miR-128 has been detected in plasma and its levels are altered in various pathological conditions [62-64], but further studies are required to better clarify the potential role of mir-128 levels as new prognostic and/or diagnostic marker in cardiovascular ischemic diseases.

\section{Abbreviations}

$\begin{array}{ll}\text { ALDH1A1: } & \text { Aldehyde dehydrogenase 1A1 } \\ \text { ARE: } & \text { Antioxidant-responsive element } \\ \text { AKR1D1: } & \text { Aldo-keto reductase family 1, member D1 } \\ \text { ALDH3A1: } & \text { Aldehyde dehydrogenase 3 family } \\ & \text { member A1 } \\ \text { CCDC53: } & \text { Coiled-coil domain containing 53 } \\ \text { HMOX-1/HO-1: } & \text { Heme oxygenase-1 } \\ \text { PCBD2: } & \text { Pterin-4 alpha-carbinolamine } \\ & \text { dehydratase 2 } \\ \text { BACH: } & \text { BTB and CNC homology } \\ \text { bZIP: } & \text { Basic-region leucine zipper } \\ \text { CHD8: } & \text { Chromo-ATPase/helicase DNA-binding } \\ & \text { protein 8 } \\ \text { CNC: } & \text { Cap 'n' collar } \\ \text { DNMT3B: } & \text { DNA methyltransferase 3 beta } \\ \text { EpRE: } & \text { Electrophile-responsive element } \\ \text { GCLC: } & \text { Glutamate-cysteine ligase catalytic } \\ \text { GST: } & \text { Glutathione S-transferase } \\ \text { HIF-1 } \alpha: & \text { Hypoxia inducible factor 1 alpha subunit } \\ \text { Keap1: } & \text { Kelch-like ECH-associated protein 1 } \\ \text { miR: } & \text { MicroRNA } \\ \text { Nrf2/NF-E2: } & \text { Nuclear factor-erythroid 2 } \\ \text { NQO1: } & \text { NAD(P):Quinone oxidoreductase 1 } \\ \text { NRF: } & \text { NF-E2 p45-related factor } \\ \text { PCR: } & \text { Polymerase chain reaction } \\ \text { PVDF: } & \text { Polyvinylidene difluoride } \\ \text { RT-qPCR: } & \text { Reverse transcription-quantitative } \\ \text { ROS: } & \text { real-time PCR } \\ \text { sMaf: } & \text { Reactive oxygen species } \\ & \text { Small Maf (musculoaponeurotic } \\ \text { UCHL1: } & \text { fibrosarcoma retrovirus transforming } \\ & \text { Ubiquitin C-terminal hydrolase L1 } \\ & \end{array}$

\section{Conflicts of Interest}

The authors declare that they have no conflicts of interest.

\section{Authors' Contributions}

Rocco Caggiano and Fabio Cattaneo contributed equally to this work.

\section{Acknowledgments}

This study was supported by the Ministero dell'Università e della Ricerca Scientifica e Tecnologica (MiUR PRIN 2009).

\section{References}

[1] K. M. Holmstrom and T. Finkel, "Cellular mechanisms and physiological consequences of redox-dependent signalling," Nature Reviews Molecular Cell Biology, vol. 15, no. 6, pp. 411-421, 2014.

[2] Y. Mikhed, A. Görlach, U. G. Knaus, and A. Daiber, "Redox regulation of genome stability by effects on gene expression, epigenetic pathways and DNA damage/repair," Redox Biology, vol. 5, pp. 275-289, 2015.

[3] L. Baird and A. T. Dinkova-Kostova, "The cytoprotective role of the Keap1-Nrf2 pathway," Archives of Toxicology, vol. 85, no. 4, pp. 241-272, 2011.

[4] J. D. Hayes and A. T. Dinkova-Kostova, "The Nrf2 regulatory network provides an interface between redox and intermediary metabolism," Trends in Biochemical Sciences, vol. 39, no. 4, pp. 199-218, 2014.

[5] C. Espinosa-Diez, V. Miguel, D. Mennerich et al., "Antioxidant responses and cellular adjustments to oxidative stress," Redox Biology, vol. 6, pp. 183-197, 2015.

[6] F. Katsuoka and M. Yamamoto, "Small Maf proteins (MafF, MafG, MafK): history, structure and function," Gene, vol. 586, no. 2, pp. 197-205, 2016.

[7] F. Katsuoka, H. Motohashi, T. Ishii, H. Aburatani, J. D. Engel, and M. Yamamoto, "Genetic evidence that small maf proteins are essential for the activation of antioxidant response element-dependent genes," Molecular and Cellular Biology, vol. 25, no. 18, pp. 8044-8051, 2005.

[8] Y. Hirotsu, F. Katsuoka, R. Funayama et al., "Nrf2-MafG heterodimers contribute globally to antioxidant and metabolic networks," Nucleic Acids Research, vol. 40, no. 20, pp. 10228 10239, 2012.

[9] G. P. Sykiotis and D. Bohmann, "Stress-activated cap'n'collar transcription factors in aging and human disease," Science Signaling, vol. 3, no. 112, article re3, 2010.

[10] K. Itoh, T. Chiba, S. Takahashi et al., "An Nrf2/small Maf heterodimer mediates the induction of phase II detoxifying enzyme genes through antioxidant response elements," Biochemical and Biophysical Research Communications, vol. 236, no. 2, pp. 313-322, 1997.

[11] A. Otsuki, M. Suzuki, F. Katsuoka et al., "Unique cistrome defined as CsMBE is strictly required for Nrf2-sMaf heterodimer function in cytoprotection," Free Radical Biology and Medicine, vol. 91, pp. 45-57, 2016.

[12] T. Q. de Aguiar Vallim, E. J. Tarling, H. Ahn et al., "MAFG is a transcriptional repressor of bile acid synthesis and metabolism," Cell Metabolism, vol. 21, no. 2, pp. 298-310, 2015.

[13] R. Fujita, M. Takayama-Tsujimoto, H. Satoh et al., "NF-E2 p45 is important for establishing normal function of platelets," Molecular and Cellular Biology, vol. 33, no. 14, pp. 2659-2670, 2013.

[14] Y. Zhang and Y. Xiang, "Molecular and cellular basis for the unique functioning of $\mathrm{Nrf1}$, an indispensable transcription factor for maintaining cell homoeostasis and organ integrity," Biochemical Journal, vol. 473, no. 8, pp. 961-1000, 2016. 
[15] F. Katsuoka, H. Yamazaki, and M. Yamamoto, "Small Maf deficiency recapitulates the liver phenotypes of Nrf1- and Nrf2-deficient mice," Genes to Cells, vol. 21, no. 12, pp. 1309-1319, 2016.

[16] Y. Zhou, H. Wu, M. Zhao, C. Chang, and Q. Lu, "The Bach family of transcription factors: a comprehensive review," Clinical Reviews in Allergy \& Immunology, vol. 50, no. 3, pp. 345-356, 2016.

[17] D. D. Zhang and M. Hannink, "Distinct cysteine residues in Keap1 are required for Keap1-dependent ubiquitination of $\mathrm{Nrf} 2$ and for stabilization of Nrf2 by chemopreventive agents and oxidative stress," Molecular and Cellular Biology, vol. 23, pp. 8137-8151, 2003.

[18] A. T. Dinkova-Kostova, R. V. Kostov, and P. Canning, "Keap1, the cysteine-based mammalian intracellular sensor for electrophiles and oxidants," Archives of Biochemistry Biophysics, vol. 617, pp. 84-93, 2017.

[19] K. Ueda, J. Xu, H. Morimoto, A. Kawabe, and S. Imaoka, "MafG controls the hypoxic response of cells by accumulating HIF-1alpha in the nuclei," FEBS Letters, vol. 582, no. 16, pp. 2357-2364, 2008.

[20] J. Sun, H. Hoshino, K. Takaku et al., "Hemoprotein Bach1 regulates enhancer availability of heme oxygenase-1 gene," The EMBO Journal, vol. 21, no. 19, pp. 5216-5224, 2002.

[21] M. Fang, L. Hutchinson, A. Deng, and M. R. Green, "Common BRAF(V600E)-directed pathway mediates widespread epigenetic silencing in colorectal cancer and melanoma," Proceedings of the National Academy of Sciences of the United States of America, vol. 113, no. 5, pp. 1250-1255, 2016.

[22] G. Stefani and F. J. Slack, "Small non-coding RNAs in animal development," Nature Reviews Molecular Cell Biology, vol. 9, no. 3, pp. 219-230, 2008.

[23] K. U. Tüfekci, M. G. Oner, R. L. Meuwissen, and S. Genç, “The role of microRNAs in human diseases," Methods in Molecular Biology, vol. 1107, pp. 33-50, 2014.

[24] D. P. Bartel, "MicroRNAs: target recognition and regulatory functions," Cell, vol. 136, no. 2, pp. 215-233, 2009.

[25] A. E. Pasquinelli, "MicroRNAs and their targets: recognition, regulation and an emerging reciprocal relationship," Nature Reviews Genetics, vol. 13, no. 4, pp. 271-282, 2012.

[26] A. K. Leung and P. A. Sharp, "MicroRNA functions in stress responses," Molecular Cell, vol. 40, no. 2, pp. 205-215, 2010.

[27] R. C. Siow and H. J. Forman, "Redox regulation of microRNAs in health and disease," Free Radical Biology and Medicine, vol. 64, pp. 1-3, 2013.

[28] D. Ayers, B. Baron, and T. Hunter, "miRNA influences in NRF2 pathway interactions within cancer models," Journal of Nucleic Acids, vol. 2015, Article ID 143636, 6 pages, 2015.

[29] A. M. Krichevsky, K. S. King, C. P. Donahue, K. Khrapko, and K. S. Kosik, "A microRNA array reveals extensive regulation of microRNAs during brain development," RNA, vol. 9, no. 10, pp. 1274-1281, 2003.

[30] J. Godlewski, M. O. Nowicki, A. Bronisz et al., "Targeting of the Bmi-1 oncogene/stem cell renewal factor by microRNA128 inhibits glioma proliferation and self-renewal," Cancer Research, vol. 68, no. 22, pp. 9125-9130, 2008.

[31] S. Venkataraman, I. Alimova, R. Fan, P. Harris, N. Foreman, and R. Vibhakar, "MicroRNA 128a increases intracellular ROS level by targeting Bmi-1 and inhibits medulloblastoma cancer cell growth by promoting senescence," PLoS One, vol. 5, no. 6, article e10748, 2010.
[32] Y. K. Adlakha and N. Saini, "MicroRNA: a connecting road between apoptosis and cholesterol metabolism," Tumour Biology, vol. 37, no. 7, pp. 8529-8554, 2016.

[33] M. Comegna, M. Succoio, M. Napolitano et al., "Identification of miR-494 direct targets involved in senescence of human diploid fibroblasts," FASEB Journal, vol. 28, no. 8, pp. 3720 3733, 2014.

[34] R. Faraonio, P. Vergara, D. D. Marzo, M. Napolitano, T. Russo, and F. Cimino, "Transcription regulation in NIH3T3 cell clones resistant to diethylmaleate-induced oxidative stress and apoptosis," Antioxidants \& Redox Signaling, vol. 8, no. 3-4, pp. 365-374, 2006.

[35] R. Faraonio, P. Vergara, D. Di Marzo et al., "p53 suppresses the Nrf2-dependent transcription of antioxidant response genes," The Journal of Biological Chemistry, vol. 281, no. 52, pp. 39776-39784, 2006.

[36] M. Succoio, M. Comegna, C. D'Ambrosio, A. Scaloni, F. Cimino, and R. Faraonio, "Proteomic analysis reveals novel common genes modulated in both replicative and stressinduced senescence," Journal of Proteomics, vol. 128, pp. 1829, 2015.

[37] G. G. Schiattarella, F. Cattaneo, G. Pironti et al., "Akap1 deficiency promotes mitochondrial aberrations and exacerbates cardiac injury following permanent coronary ligation via enhanced mitophagy and apoptosis," PLoS One, vol. 11, no. 5, article e0154076, 2016.

[38] F. Cattaneo, M. Parisi, T. Fioretti, D. Sarnataro, G. Esposito, and R. Ammendola, "Nuclear localization of formyl-peptide receptor 2 in human cancer cells," Archives of Biochemistry and Biophysics, vol. 603, pp. 10-19, 2016.

[39] K. J. Livak and T. D. Schmittgen, "Analysis of relative gene expression data using real-time quantitative PCR and the 2(-delta delta C(T))," Methods, vol. 25, no. 4, pp. 402408, 2001.

[40] G. Esposito, G. G. Schiattarella, C. Perrino et al., "Dermcidin: a skeletal muscle myokine modulating cardiomyocyte survival and infarct size after coronary artery ligation," Cardiovascular Research, vol. 107, no. 4, pp. 431-441, 2015.

[41] P. Siengdee, N. Trakooljul, E. Murani, M. Schwerin, K. Wimmers, and S. Ponsuksili, "MicroRNAs regulate cellular ATP levels by targeting mitochondrial energy metabolism genes during $\mathrm{C} 2 \mathrm{C} 12$ myoblast differentiation," PLoS One, vol. 10, no. 5, article e0127850, 2015.

[42] L. Shi, B. Zhou, P. Li et al., "MicroRNA-128 targets myostatin at coding domain sequence to regulate myoblasts in skeletal muscle development," Cellular Signalling, vol. 27, no. 9, pp. 1895-1904, 2015.

[43] B. Bodega, F. Marasca, V. Ranzani et al., "A cytosolic Ezh1 isoform modulates a PRC2-Ezh1 epigenetic adaptive response in postmitotic cells," Nature Structural \& Molecular Biology, vol. 24, no. 5, pp. 444-452, 2017.

[44] S. A. Agrawal, D. Anand, A. D. Siddam et al., "Compound mouse mutants of bZIP transcription factors Mafg and Mafk reveal a regulatory network of non-crystallin genes associated with cataract," Human Genetics, vol. 134, no. 7, pp. 717-735, 2015.

[45] W. Li, S. Yu, T. Liu et al., "Heterodimerization with small Maf proteins enhances nuclear retention of Nrf2 via masking the NESzip motif," Biochimica et Biophysica Acta (BBA)-Proteins and Proteomics, vol. 1783, no. 10, pp. 1847-1856, 2008. 
[46] R. Faraonio, F. Pane, M. Intrieri, T. Russo, and F. Cimino, "In vitro acquired cellular senescence and aging-specific phenotype can be distinguished on the basis of specific mRNA expression," Cell Death and Differentiation, vol. 9, no. 8, pp. 862-864, 2002.

[47] X. Cheng, C. H. Ku, and R. C. Siow, "Regulation of the Nrf2 antioxidant pathway by microRNAs: new players in micromanaging redox homeostasis," Free Radical Biology and Medicine, vol. 64, pp. 4-11, 2013.

[48] S. Kurinna and S. Werner, "NRF2 and microRNAs: new but awaited relations," Biochemical Society Transactions, vol. 43, no. 4, pp. 595-601, 2015.

[49] C. Zhang, L. Shu, and A. N. Kong, "MicroRNAs: new players in cancer prevention targeting $\mathrm{Nrf} 2$, oxidative stress and inflammatory pathways," Current Pharmacology Reports, vol. 1, no. 1, pp. 21-30, 2015.

[50] Y. Guo, S. Yu, C. Zhang, and A. N. Kong, "Epigenetic regulation of Keap1-Nrf2 signaling," Free Radical Biology and Medicine, vol. 88, Part B, pp. 337-349, 2015.

[51] X. Sun, H. Zuo, C. Liu, and Y. Yang, "Overexpression of miR-200a protects cardiomyocytes against hypoxia-induced apoptosis by modulating the kelch-like ECH-associated protein 1-nuclear factor erythroid 2-related factor 2 signaling axis," International Journal of Molecular Medicine, vol. 38, no. 4, pp. 1303-1311, 2016.

[52] J. Y. Chan and S. H. Chan, "Activation of endogenous antioxidants as a common therapeutic strategy against cancer, neurodegeneration and cardiovascular diseases: a lesson learnt from DJ-1," Pharmacology \& Therapeutics, vol. 156, pp. 69$74,2015$.

[53] K. Kanninen, R. Heikkinen, T. Malm et al., "Intrahippocampal injection of a lentiviral vector expressing Nrf2 improves spatial learning in a mouse model of Alzheimer's disease," Proceedings of the National Academy of Sciences of the United States of America, vol. 106, pp. 16505-16510, 2009.

[54] R. T. Kolamunne, I. H. Dias, A. B. Vernallis, M. M. Grant, and H. R. Griffiths, "Nrf2 activation supports cell survival during hypoxia and hypoxia/reoxygenation in cardiomyoblasts; the roles of reactive oxygen and nitrogen species," Redox Biology, vol. 1, pp. 418-426, 2013.

[55] K. J. Liang, K. T. Woodard, M. A. Weaver, J. P. Gaylor, E. R. Weiss, and R. J. Samulski, "AAV-Nrf2 promotes protection and recovery in animal models of oxidative stress," Molecular Therapy, vol. 25, no. 3, pp. 765-779, 2017.

[56] V. Malec, O. R. Gottschald, S. Li, F. Rose, W. Seeger, and J. Hänze, "HIF-1 alpha signaling is augmented during intermittent hypoxia by induction of the Nrf2 pathway in NOX1-expressing adenocarcinoma A549 cells," Free Radical Biology and Medicine, vol. 48, no. 12, pp. 1626-1635, 2010.

[57] R. B. Donker, J. F. Mouillet, D. M. Nelson, and Y. Sadovsky, "The expression of Argonaute2 and related microRNA biogenesis proteins in normal and hypoxic trophoblasts," Molecular Human Reproduction, vol. 13, pp. 273-279, 2007.

[58] X. C. Zeng, L. Li, H. Wen, and Q. Bi, "MicroRNA-128 inhibition attenuates myocardial ischemia/reperfusion injury-induced cardiomyocyte apoptosis by the targeted activation of peroxisome proliferator-activated receptor gamma," Molecular Medicine Reports, vol. 14, pp. 129-136, 2016.

[59] J. Loscalzo, Harrison's Cardiovascular Medicine, McGraw-Hill Medical, New York, 2010.
[60] Y. Huang, W. Li, Z. Y. Su, and A. N. Kong, "The complexity of the Nrf2 pathway: beyond the antioxidant response," The Journal of Nutritional Biochemistry, vol. 26, no. 12, pp. 1401-1413, 2015.

[61] D. E. Handy and J. Loscalzo, "Responses to reductive stress in the cardiovascular system," Free Radical Biology and Medicine, vol. 109, pp. 114-124, 2017.

[62] L. Zhuang, L. Xu, P. Wang, and Z. Meng, "Serum miR-128-2 serves as a prognostic marker for patients with hepatocellular carcinoma," PLoS One, vol. 10, no. 2, article e0117274, 2015.

[63] J. Sun, K. Liao, X. Wu, J. Huang, S. Zhang, and X. Lu, "Serum microRNA-128 as a biomarker for diagnosis of glioma," International Journal of Clinical and Experimental Medicine, vol. 8, no. 1, pp. 456-463, 2015.

[64] J. Vistbakka, I. Elovaara, T. Lehtimäki, and S. Hagman, "Circulating microRNAs as biomarkers in progressive multiple sclerosis," Multiple Sclerosis Journal, vol. 23, no. 3, pp. 403-412, 2017. 


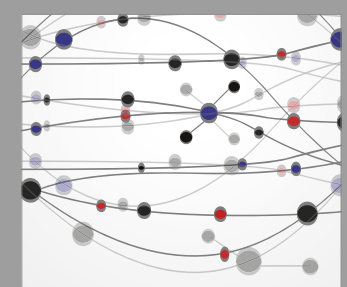

The Scientific World Journal
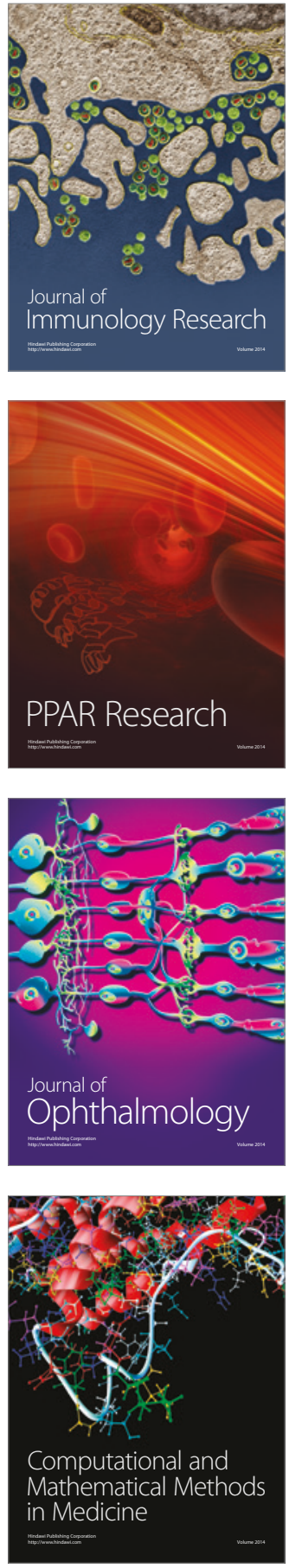

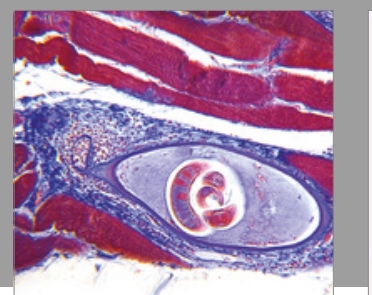

Gastroenterology Research and Practice
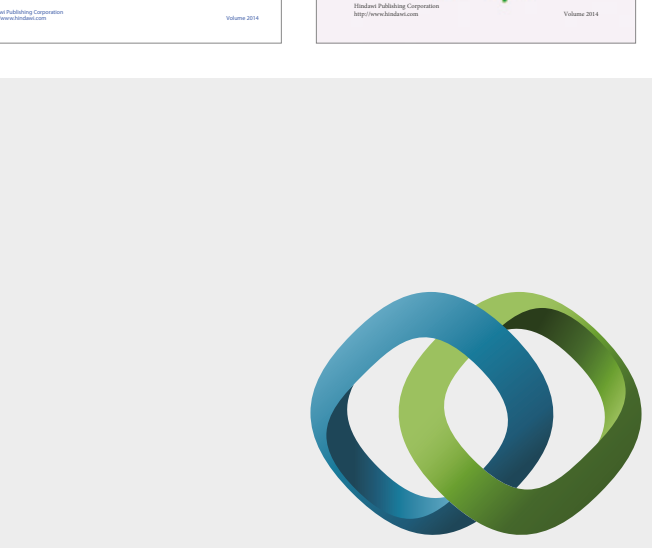

\section{Hindawi}

Submit your manuscripts at

https://www.hindawi.com
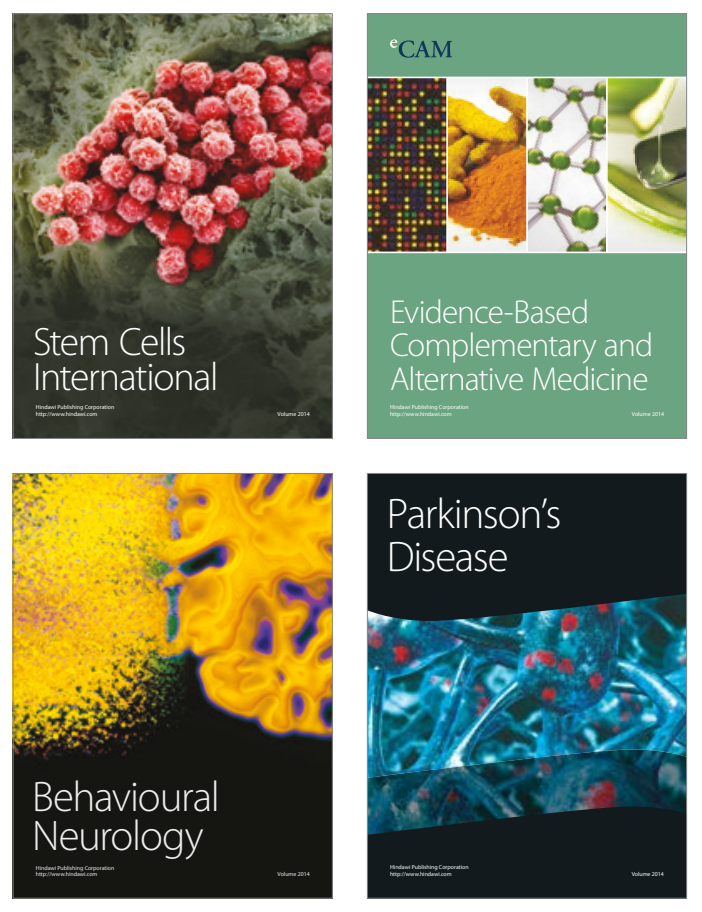
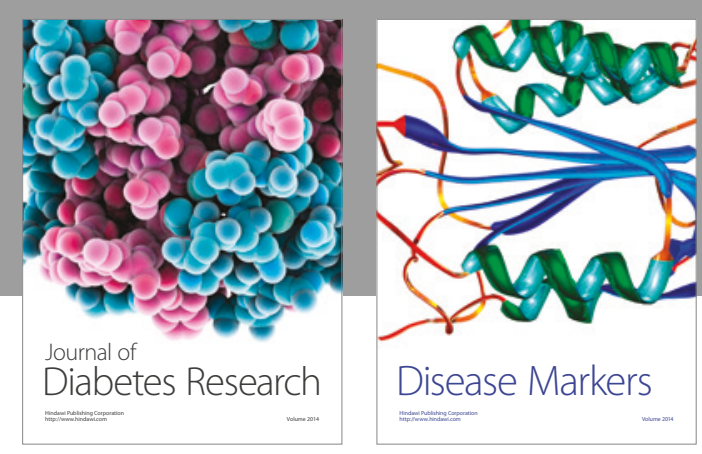

Disease Markers
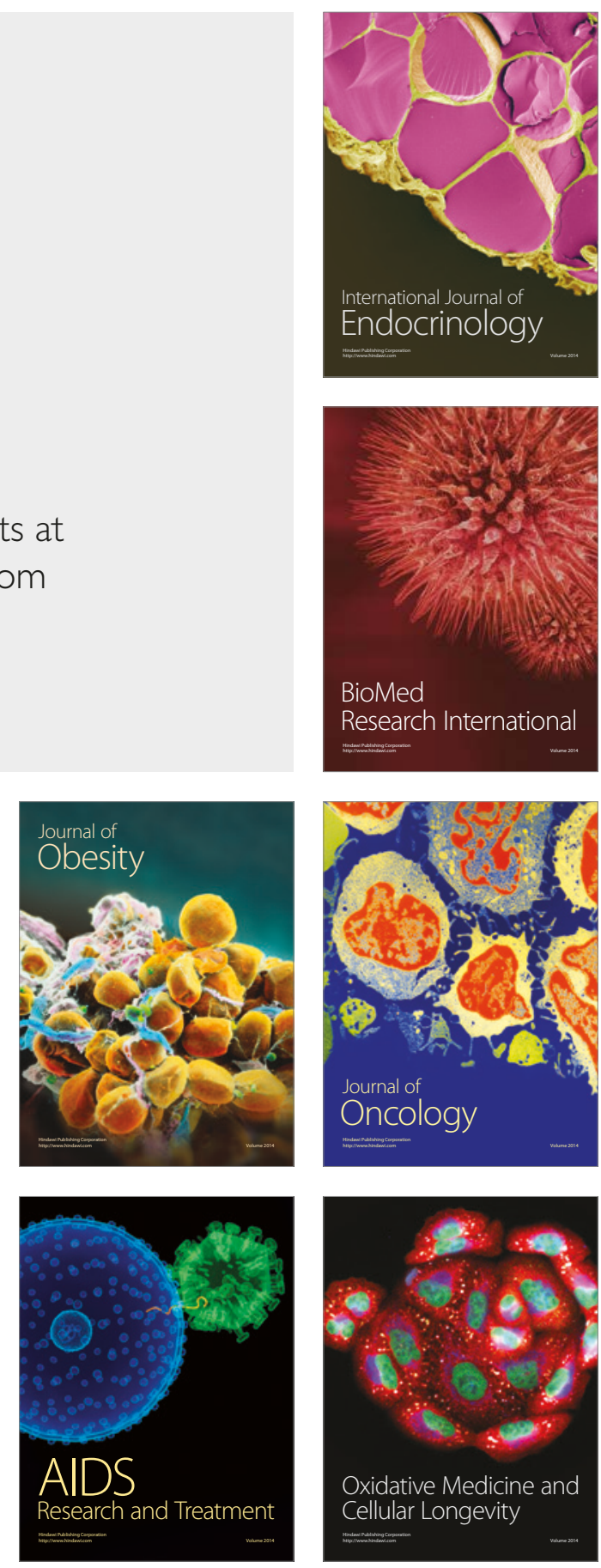